\title{
TIPOS DO HERBÁRIO PRISCO BEZERRA - EAC
}

\author{
Maria Stela Bezerra da Silva' \\ Cláudia Regina Chagas Pompeu' \\ Francisca Simões Cavalcante ${ }^{2}$ \\ Afrânio Fernandes ${ }^{3}$
}

\begin{abstract}
RESUMO - Com o presente trabalho pretende-se contribuir para a divulgação dos tipos do Herbário Prisco Bezerra - EAC. Um levantamento realizado no acervo do Herbário revelou o registro de dez tipos. Sete deles pertencentes à familia Leguminosae distribuídas entre as subfamílias Caesalpinioideae: Peltogyne crenulata, Senna dardanoi, Moldenhawera acuminata, Chamaecrista duckeana, Papilionoideae: Aeschynomene monteiroi, Cranocarpus gracilis e Mimosoideae: Mimosa niomarlei; dois, pertencentes à família Erythroxylaceae. As espécies Erythroxylum tianguanum e Erythroxylum bezerrae e um à família Euphorbiaceae: Dalechampia fernandesï recentemente descrita por G. Webster. Os tipos são apresentados na seqüência das indicações: nome das famílias, seguido do epíteto específico, habitat, com a transferência de todas as informações mencionadas na ficha de exsicata do material, bem como, a fotografia correspondente. Citam-se também alguns aspectos característicos das espécies, para melhor visualização dos detalhes fotográficos.
\end{abstract}

Palavras-chave: Herbário, tipos

ABSTRACT - The present paper repasts the type specimens in the Herbário Prisco Bezerra (University of Ceará). The Herbário has ten type, of which seven are Leguminosae: Peltoqyne crenulata, Senna dardanoi, Moldenhawera acuminata Chamaecrista duckeana, Aeschynomene monteiroi, Cranocarpus gracilis, Mimosa niomarlei, two the Erythroxylaceae: Erythroxylum tianquanum and Erythroxylum bezerrae and one Euphorbiaceae: Dalechampia fernandesii. Additional informations for these exsicata is presented.

Key-words: Herbarium typi.

\section{Introdução}

A divulgação das espécies que compõem o acervo de um herbário 'é

1 - Monitoras das Disciplinas Sistemática Vegetal - Departamento de Biologia - UFC.

2 - Eng. Agrônoma, Curadora do Herbário Prisco Bezerra - EAC - Departamento de Biologia - UFC.

3 - Prof. Emérito - Departamento de Biologia - UFC. 
fundamental para intensificação do intercâmbio entre eles. O desenvolvimento das pesquisas botânicas conta com esse veículo básico para ampliar o conhecimento acerca da composição florística das diversas regiões do globo terrestre.

Pretende-se, portanto, contribuir com os estudiosos do assunto, divulgando-se os tipos do Herbário Prisco Bezerra.

\section{Material e método}

O material utilizado neste levantamento encontra-se no Herbário Prisco Bezerra. Trata-se dos tipos ali depositados, que serviram para a descrição das respectivas espécies. Foram reunidas na Tabela 1, para melhor observação do conjunto de dados, o nome da família, o epíteto específico seguido da indicação numérica da fotografia correspondente as informações contidas nas fichas das exsicatas referentes à coleta botânica, bem como o nome do autor responsável pela descrição da espécie, conforme referências bibliográficas.

\section{Resultados}

O levantamento revelou o registro de 10 (dez) tipos. Dentre os tipos identificados, sete foram encontrados no Estado do Ceará e três nos Estados de Maranhão, Piauí e Mato Grosso do Sul. Estão distribuídos nas seguintes familias: L'suminosae, Erythroxylaceae e Euphorbiaceae. Na família Leguminosae estão: Peltogyne crenulata A. Fernandes, (Figura 9) Senna dardanoi A. Fernandes \& P. Bezerra (Figura 1), Moldenhawera acuminata A. Fernandes \& P. Bezerra (Figura 4) e Chcmaecrista duckeana P. Bezerra \& A. Fernandes (Figura 6), pertencentes à subfamília Caesalpinioideae; Atschynomene monteiroi A. Fernandes \& P. Bezerra (Figura 5) e Cianocarpus gracilis A. Fernandes \& P. Bezerra (Figura 2), à subfamília Papilionoideae; e Mirrosa niomarlei A. Fernandes (Figura 3), à subfamília Mimosoideae. Dentro da família Erythroxylaceae estão: Erythroxylum tianguanum Plowman (Figura 7) e Erythroxyluma bezerrae Plowman (Figura 8), enquanto que na família Euphorbiaceae está a espécie Dalechampia fernandesii G. Webster.

Com relação a esses tipos podemos enfatizar alguns detalhes de formação do epíteto específico. A espécie Senna dardanoi tem este nome em homenagem ao botânico Dárdano de Andrade Lima. A espécie Dalechampia fernandesii foi uma homenagem prestada por G. Webster ao botânico Afrânio Fernandes, então curador do Herbário Prisco Bezerra. A Aeschynomene monteiroi recebeu este nome em homenagem a outro renomado botânico Honório Monteiro Filho, enquanto que Erythroxylum bezerrae homenageia Dr. Prisco Bezerra, eminente Senhor Botânico da UFC. A Erythroxulum tianguanum re- 
cebeu este nome em referência à cidade serrana de Tianguá, conhecido município cearense.

\section{Referências bibliográficas}

BARNEBY, R.C. \& H.S. IRWIN. 1982. The american Cassinae: a synoptical revision of Leguminosae tribe Cassinae subtribe Cassinae in the world. Mem. New York Bot. Gdn. 35(2):918.

FERNANDES, A. \& P. BEZERRA. 1984. Uma nova espécie de Fabaceae do gênero Senna Mill. Bradea 4(8):49-54.

FERNANDES, A. 1968. Mimosa no Ceará - nova espécie. In: Congresso Brasileiro de Botânica, 19., Fortaleza.

FERNANDES, A. \& P. BEZERRA. 1979. O gênero Cranocarpus (Leguminosae) uma nova espécie. Bradea 2(5):329-38.

FERNANDES, A. \& P. BEZERRA. 1982. O gênero Moldenhawera (Leguminosae-Caesalpinioidea) no Brasil - uma espécie. In: Congresso Nacional de Botânica, 32.: Teresina-PI, p. 58-62.

FERNANDES, A. 1988. Uma nova espécie de Peltogyne Vogel (Fabaceae): Peltogyne crenulata. In: Congresso Nacional de Botânica 39., Belém.

PLOWMAN, T. 1986. Four new species of Erythroxylum (Erythroxylaceae) from Northeastern Brazil. Brittonia, 38(3):189-200.

WEBSTER, G. L. 1989. Three new species of Dalechampia (Euphorbiaceae) from Brazil. Brittonia, 41(1):1-9, 1989. 
SILVA et al.

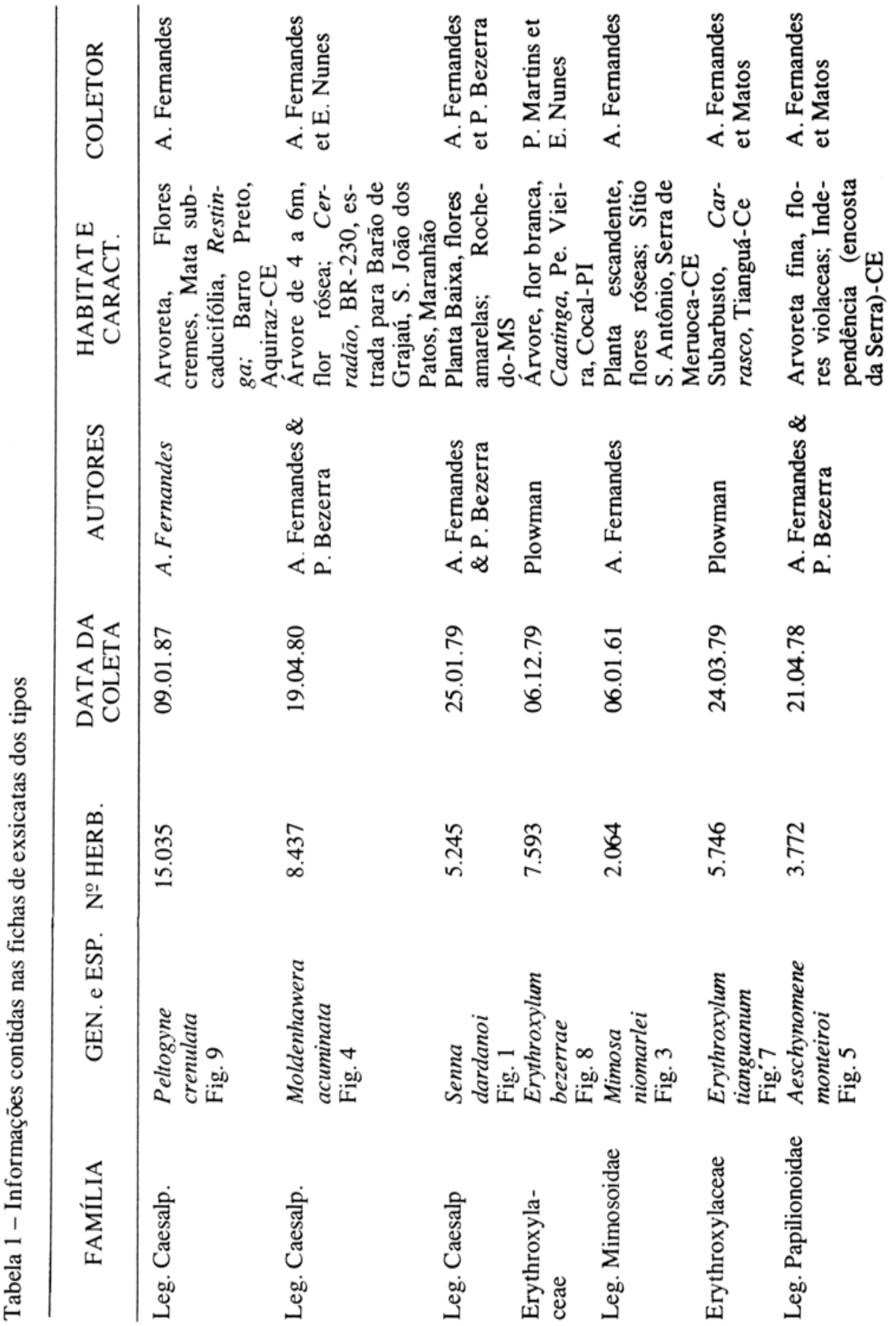



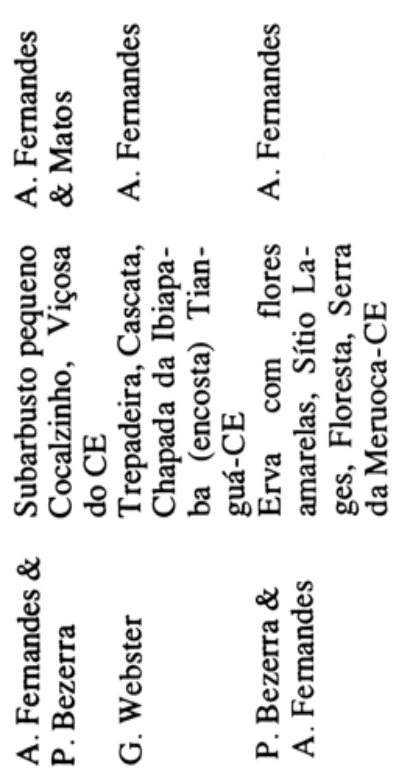

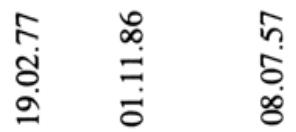

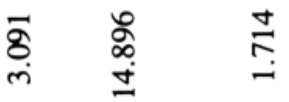
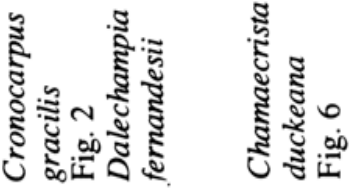

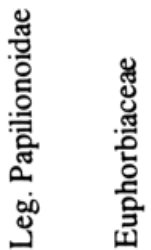

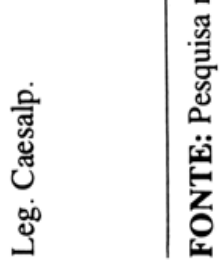




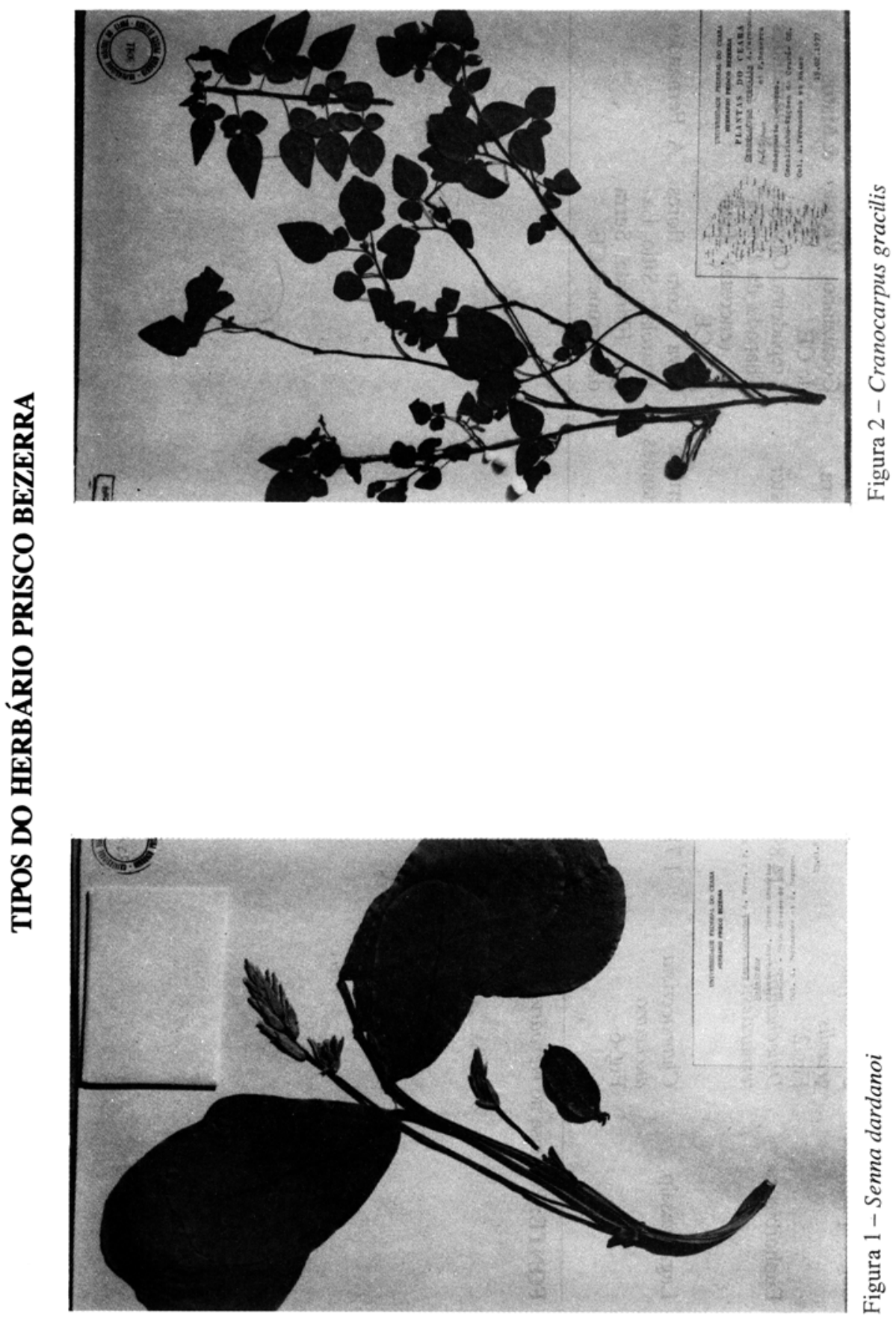


Tipos do herbário Prisco Bezerra - EAC

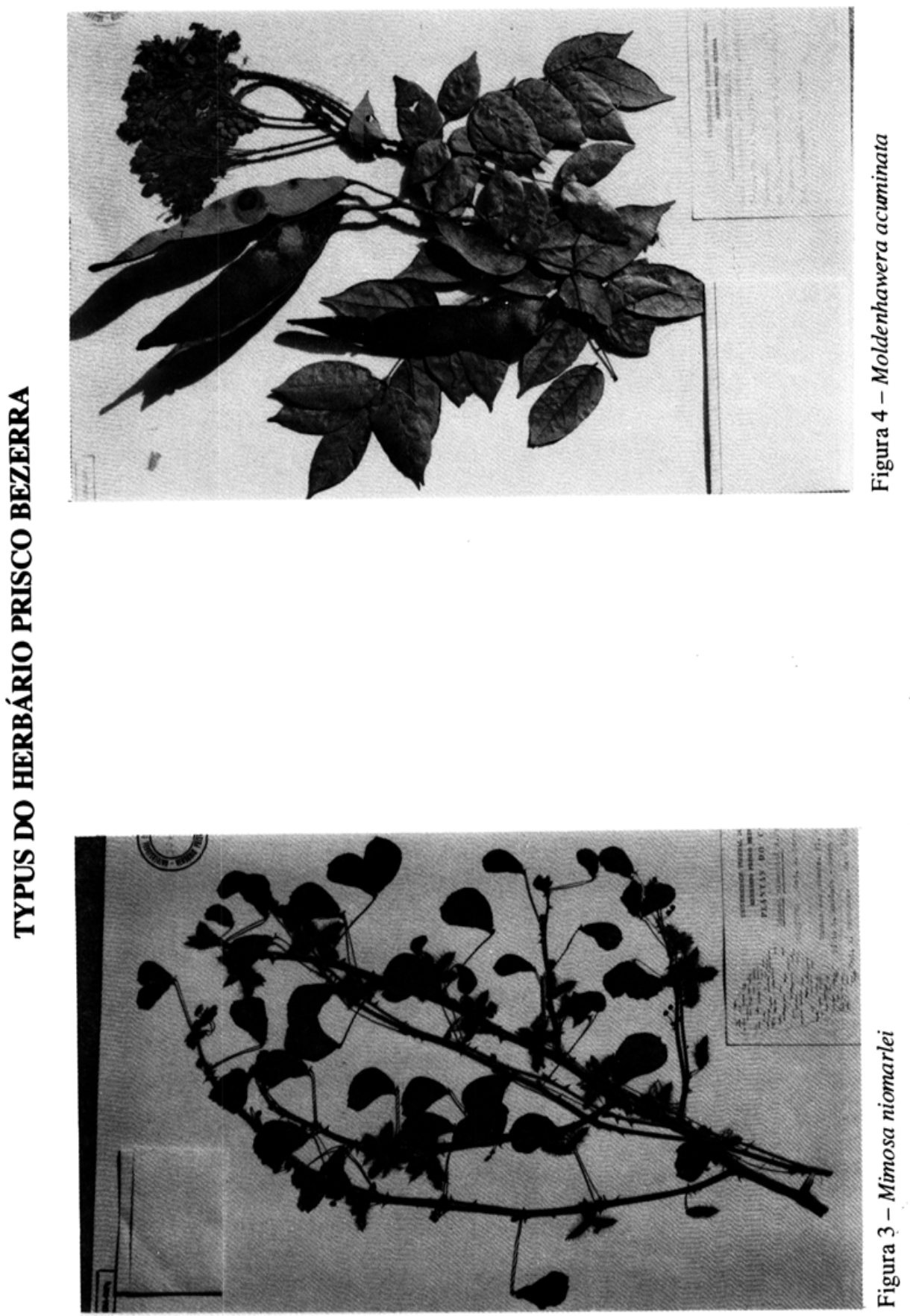




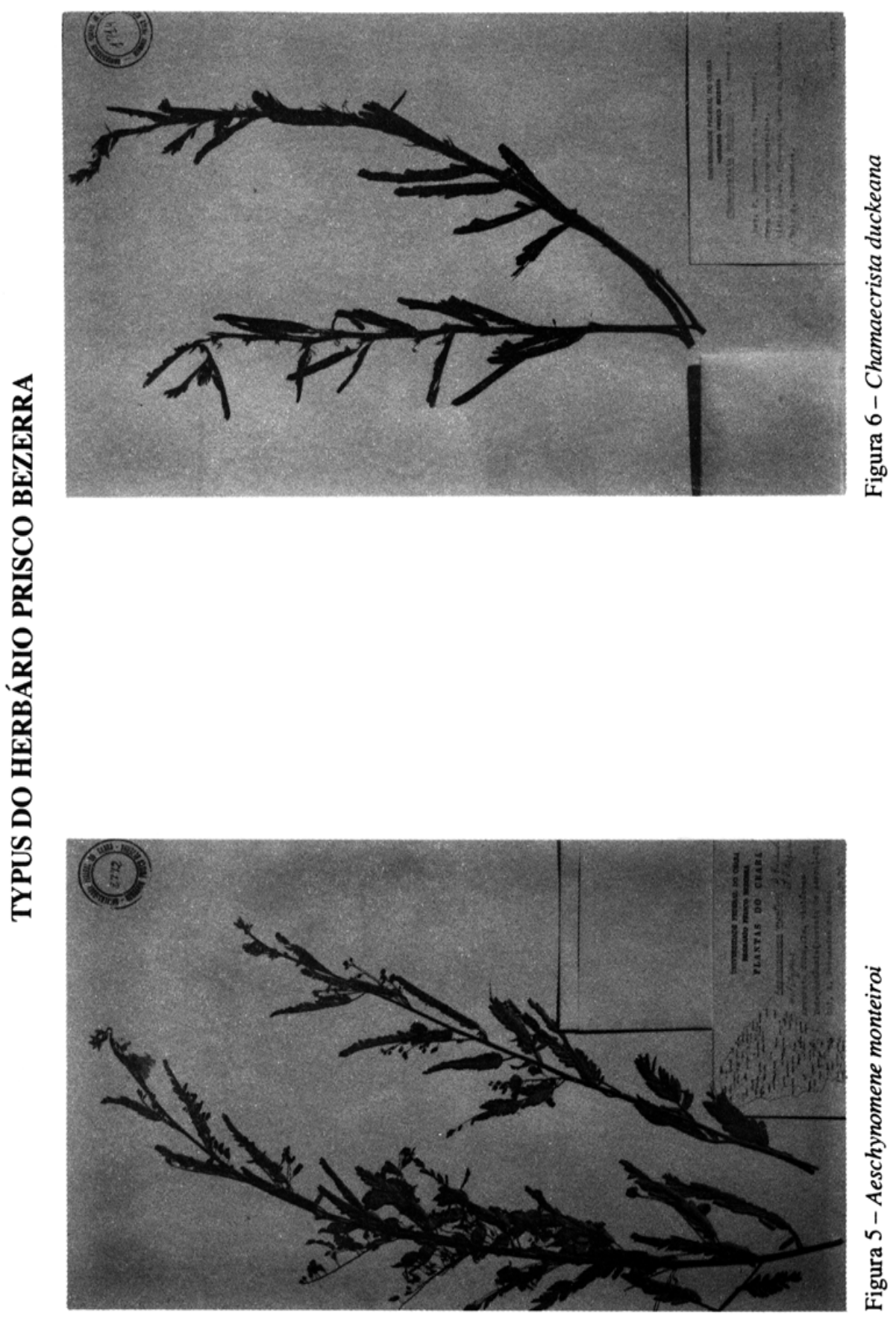




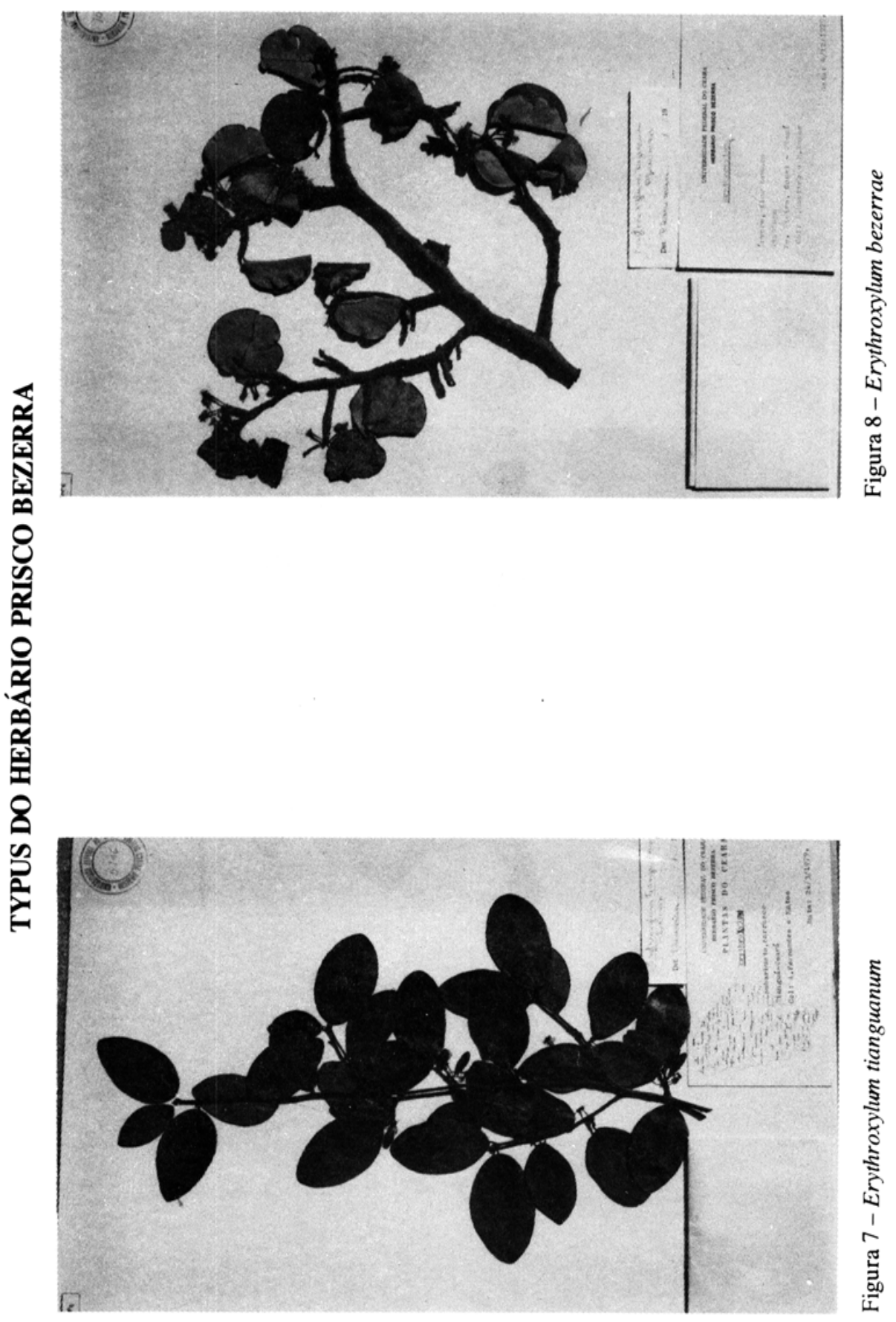




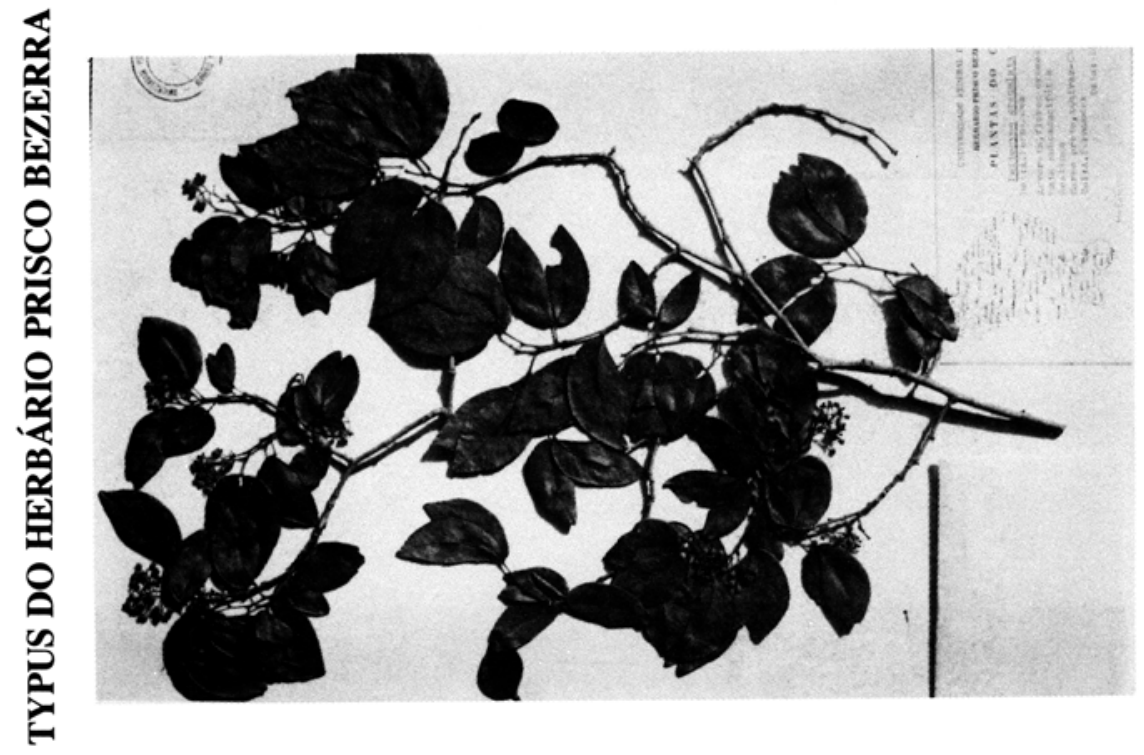

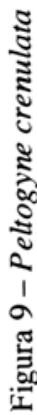

